

\title{
Hybrid Sparse Regularization for Magnetic Resonance Spectroscopy
}

Andrea Laruelo, Lotfi Chaari, Hadj Batatia, Soleakhena Ken, Ben Rowland, Anne Laprie, Jean-Yves Tourneret

\section{To cite this version:}

Andrea Laruelo, Lotfi Chaari, Hadj Batatia, Soleakhena Ken, Ben Rowland, et al.. Hybrid Sparse Regularization for Magnetic Resonance Spectroscopy. IEEE Engineering in Medicine and Biology Society Conference - EMBC 2013, Jul 2013, Osaka, Japan. pp. 6768-6771. hal-01148277

\section{HAL Id: hal-01148277 https://hal.science/hal-01148277}

Submitted on 4 May 2015

HAL is a multi-disciplinary open access archive for the deposit and dissemination of scientific research documents, whether they are published or not. The documents may come from teaching and research institutions in France or abroad, or from public or private research centers.
L'archive ouverte pluridisciplinaire $\mathbf{H A L}$, est destinée au dépôt et à la diffusion de documents scientifiques de niveau recherche, publiés ou non, émanant des établissements d'enseignement et de recherche français ou étrangers, des laboratoires publics ou privés. 




\section{Open Archive TOULOUSE Archive Ouverte (OATAO)}

OATAO is an open access repository that collects the work of Toulouse researchers and makes it freely available over the web where possible.

This is an author-deposited version published in : http://oatao.univ-toulouse.fr/ Eprints ID : 12970

To link to this article : DOI :10.1109/EMBC.2013.6611110 URL : http://dx.doi.org/10.1109/EMBC.2013.6611110

To cite this version : Laruelo, Andrea and Chaari, Lotfi and Batatia, Hadj and Ken, Soleakhena and Rowland, Ben and Laprie, Anne and Tourneret, Jean-Yves Hybrid Sparse Regularization for Magnetic Resonance Spectroscopy. (2013) In: IEEE Engineering in Medicine and Biology Society Conference - EMBC 2013, 3 July 2013 - 7 July 2013 (Osaka, Japan).

Any correspondance concerning this service should be sent to the repository administrator: staff-oatao@,listes-diff.inp-toulouse.fr 


\title{
Hybrid sparse regularization for Magnetic Resonance Spectroscopy
}

\author{
Andrea Laruelo ${ }^{1,2}$, Lotfi Chaari ${ }^{2}$, Hadj Batatia ${ }^{2}$, Soleakhena Ken ${ }^{1}$, \\ Ben Rowland ${ }^{1}$, Anne Laprie ${ }^{1,3,4}$ and Jean-Yves Tourneret ${ }^{2}$
}

\begin{abstract}
Magnetic resonance spectroscopy imaging (MRSI) is a powerful non-invasive tool for characterising markers of biological processes. This technique extends conventional MRI by providing an additional dimension of spectral information describing the abnormal presence or concentration of metabolites of interest. Unfortunately, in vivo MRSI suffers from poor signal-to-noise ratio limiting its clinical use for treatment purposes. This is due to the combination of a weak MR signal and low metabolite concentrations, in addition to the acquisition noise. We propose a new method that handles this challenge by efficiently denoising MRSI signals without constraining the spectral or spatial profiles. The proposed denoising approach is based on wavelet transforms and exploits the sparsity of the MRSI signals both in the spatial and frequency domains. A fast proximal optimization algorithm is then used to recover the optimal solution. Experiments on synthetic and real MRSI data showed that the proposed scheme achieves superior noise suppression (SNR increase up to $60 \%$ ). In addition, this method is computationally efficient and preserves data features better than existing methods.
\end{abstract}

\section{INTRODUCTION}

MRSI is a non-invasive technique that has become a valuable tool to characterize metabolic processes and neurological disorders such as brain tumor [1]. It was initially developed for examination of human brain tumours, and its use has been extended for examination of prostate and breast cancers [2]. Its complementary use with MRI provides useful information on tumour characteristics, progression and response to treatment. Single Voxel Spectroscopy (SVS) techniques identifying abnormal levels of various metabolites have been used for diagnosis purposes for many years and in a variety of illnesses. More recently, much more interest has been developed to Chemical Shift Imaging (CSI) techniques, which allow the generation of "metabolic maps" showing the variation in metabolite concentrations over a large volume [3]. Unfortunately, this spatial information comes at the cost of a reduced signal-to-noise ratio (SNR), due to a reduced scan time for each voxel. This makes accurate quantification of metabolite signals extremely challenging. Therefore, improving the SNR is a key factor in achieving clinical utility for CSI techniques. Signal averaging is a standard technique to improve SNR. However, this approach is often not practical because of long acquisition times. Another popular method is to apply apodizing filters but this

The research leading to these results has received funding from the European Union Seventh Framework Programme (FP7-PEOPLE-2011-ITN) under grant agreement PITN-GA-2011290148.

${ }^{1}$ Institut Claudius Regaud, Toulouse, F-31052 France lastname.firstname@claudiusregaud. fr

${ }^{2}$ Univ. of Toulouse, IRIT - INP-ENSEEIHT, France firstname. lastnamedenseeiht. fr

${ }^{3}$ Université Toulouse III Paul Sabatier, Toulouse, F-31000 France

${ }^{4}$ INSERM UMR825, Toulouse, F-31024, France often compromises the spectral resolution of the data [4]. Many of the algorithms dedicated to MRSI data processing rely heavily on spectral constraints and struggle with very noisy signals. These methods incorporate constraints on the spectral profile, either by using explicit parametric models or by assuming particular line-shape characteristics [5][7]. Consequently, approaches described hereabove tend to bias the interpretation of the measured signal towards the prior assumptions. The limited ability of these models to capture the spatial variations exhibited by in vivo scans, especially in the context of lesions, make them ineffective in practical applications. In addition, these methods are often sensitive to several parameters. It is desirable then to have a method able to suppress noise in MRSI signals in a nonconstrained way while preserving the spectral features. Since there might be applications where prior information can be reliably incorporated, such a method should also allow natural extensions that preserve this possibility.

In this paper, we investigate the sparse regularization issue to address the limitations mentioned above. We propose a novel method for MRSI denoising, which accounts for signal regularity across the spectral dimension without altering the spectral resolution, but also across the spatial dimension. The proposed approach relies on sparsity promoting priors using wavelet transforms, a key signal/image tool that has been successfully used in a wide spectrum of image processing applications, especially in the biomedical field [8]-[10]. We then use a fast optimization algorithm to deal with the convex optimality criterion which is not differentiable. For doing so, proximal algorithms have been investigated, a family of fast convex optimization algorithms that have also been widely used in signal/image processing [9], [11].

The remainder of this paper is organized as follows. Section II formulates the MRSI denoising problem. The proposed sparse regularization method is detailed in Section III. Illustrations on synthetic and real data are presented in Section IV. Finally, conclusions and perspectives are drawn in Section V.

\section{MRSI DENOISING}

MRSI signals are not directly available, but only measured through some physical laws relating the sought signal to the measurements. Let $\boldsymbol{S}$ and $\boldsymbol{Z}$ be the target and observed MRSI signals, respectively, corresponding to a $2 \mathrm{D}$ slice involving $N$ spectral points. Denoting the 2D spatial position by $r$, $\boldsymbol{S}$ can be written as $\boldsymbol{S}=\left(\boldsymbol{s}^{\boldsymbol{r}}\right)_{\boldsymbol{r} \in \boldsymbol{R}}=\left(\boldsymbol{y}^{f}\right)_{1 \leq f \leq N}$, where $\boldsymbol{R}$ involves all spatial positions, the $N \times 1$ vector $\boldsymbol{s}^{\boldsymbol{r}}$ denotes the spectrum at voxel $\boldsymbol{r}$, and $\boldsymbol{y}^{f}$ the 2D image of size $N_{r} \times N_{c}$ related to frequency $f$. The observation model is:

$$
\boldsymbol{Z}=\boldsymbol{S}+\boldsymbol{n}
$$


where $\boldsymbol{n}$ is an additive complex-valued Gaussian noise of diagonal covariance matrix $\Sigma$ which has to be estimated.

Existing methods recover $S$ from Eq. (1) either by using general schemes where the inherent spectro-spatial dimensions are not explored, or by assuming restrictive models that are not able to deal with the variability of MRSI in vivo data. These are some of the drawbacks of the current methods, like the widely used linear-shift invariant filters, the standard wavelet shrinkage or the method presented in [5]. The approach proposed in this paper estimates $\boldsymbol{S}$ from Eq. (1) by adopting a sparse regularization scheme that is less constrained than the previous approaches, is able to capture the spatio-spectral nature of MRSI data and keeps open the feasibility of incorporating prior knowledge into the model.

\section{SPATIAL SPECTRAL MRSI DENOISING}

\section{A. Motivation}

Quantification of MRSI signals is a challenging problem. One of the key factors to achieve the clinical utility of MR spectroscopy is the SNR improvement for this type of data. Current MRSI denoising techniques rely heavily on spectral constraints and thus are not able to describe the spatial variations exhibited by in vivo data. The method presented here is able to overcome these limitations by removing the spurious spatio-spectral irregularities while preserving spatial and spectral resolutions. In addition, unlike the existing regularizations schemes, this method relies on an efficient optimization algorithm and it is not subject to local optima.

\section{B. Optimization criterion}

At a given spectral frequency $f$, the 2D complex-valued image $\boldsymbol{y}^{f}$ of size $N_{r} \times N_{c}$ can be seen as an element of the Euclidean space $\mathbb{C}^{K}$ with $K=N_{r} \times N_{c}$ endowed with the standard inner product $\langle\cdot \mid \cdot\rangle$ and norm $\|\cdot\|$. We employ a dyadic 2D orthonormal wavelet decomposition operator $T$ over $j_{\max }^{1}$ resolution levels. The coefficient field resulting from the wavelet decomposition of a target image $\boldsymbol{y}^{f}$ is defined as $\zeta^{f}=\left(\boldsymbol{\zeta}_{a}^{f},\left(\boldsymbol{\zeta}_{o, j}^{f}\right)_{o \in \mathbb{O}, 1 \leq j \leq j_{\max }^{1}}\right)$ with $o \in$ $\mathbb{O}=\{0,1\}^{2} \backslash\{(0,0)\}, \boldsymbol{\zeta}_{a}^{f}=\left(\zeta_{a, k}^{f}\right)_{1 \leq k \leq K_{j_{\max }}}$ and $\boldsymbol{\zeta}_{o, j}^{f}=$ $\left(\zeta_{o, j, k}^{f}\right)_{1 \leq k \leq K_{j}}$ where $K_{j}=K 2^{-2 j}$ is the number of wavelet coefficients in a given subband at resolution $j$ (by assuming that $Y_{1}$ and $Y_{2}$ are multiples of $2^{j_{\max }}$. Adopting such a notation, the wavelet coefficients have been re-indexed so that $\zeta_{a}^{f}$ denotes the approximation coefficient vector at the resolution level $j_{\max }$, while $\zeta_{o, j}^{f}$ denotes the detail coefficient vector at the orientation $o$ and resolution level $j$.

On the other hand, the spectrum $s^{r}$ of size $N$ at spatial position $\boldsymbol{r}$ can be seen as an element of the Euclidean space $\mathbb{C}^{N}$ endowed with the standard inner product and norm. We denote here by $F$ the dyadic 1D orthonormal wavelet decomposition operator over $j_{\max }^{2}$ resolution levels. The coefficient field corresponding to the spectrum $s^{r}$ is then denoted by $\zeta^{\boldsymbol{r}}=\left(\boldsymbol{\zeta}_{a}^{\boldsymbol{r}},\left(\boldsymbol{\zeta}_{d, j}^{\boldsymbol{r}}\right)_{1 \leq j \leq j_{\max }^{2}}\right)$, where the subscripts $a$ and $d$ denote approximation and detail subbands, respectively.

In order to reduce the search space of $\boldsymbol{S}$ to solutions having fewer irregularities both in the spatial and spectral dimensions, we propose here to introduce two regularization terms. The first one describes the 2D spatial prior knowledge about the wavelet coefficients of the target solution. This first regularization term is defined as:

$$
\begin{aligned}
g(\boldsymbol{S})= & \sum_{f=1}^{N}\left[\sum_{k=1}^{K_{j_{\max }^{1}}} \Phi_{\mu_{a}, \alpha_{a}}^{p}\left(\left(T \boldsymbol{y}^{f}\right)_{a, k}\right)\right. \\
& \left.+\sum_{o \in \mathbb{O}} \sum_{j=1}^{j_{\max }^{1}} \sum_{k=1}^{K_{j}} \Phi_{\mu_{o}^{j}, \alpha_{o}^{j}}^{p}\left(\left(T \boldsymbol{y}^{f}\right)_{o, j, k}\right)\right]
\end{aligned}
$$

where $\forall \xi \in \mathbb{C}, \Phi_{\mu, \alpha}^{p}(\xi)=\alpha|\xi-\mu|_{p}^{p}, \mu_{o}^{j} \in \mathbb{C}, \alpha_{o}^{j} \in \mathbb{R}_{+}$and $p \geq 1$ (similarly $\mu_{a}$ and $\alpha_{a}$ ). Due to the shape parameter $p$, this regularization term keeps a compromise between sparsity ( $p \sim 1)$ and smoothness $(p \sim 2)$ of the wavelet coefficients of the 2D image $\boldsymbol{y}^{f}$. The second regularization term allows reducing high spectral discontinuities (especially close to the metabolite peaks) between adjacent frequency bands at a voxel $\boldsymbol{r}$. This regularization is made through the penalization of the wavelet coefficients of the 1D spectra $s^{r}$ :

$$
\begin{array}{r}
h(\boldsymbol{S})=\sum_{\boldsymbol{r} \in \boldsymbol{R}}\left[\sum_{k=1}^{K_{j_{\max }^{2}}} \Phi_{\eta_{a}, \lambda_{a}}^{\beta}\left(\left(F \boldsymbol{s}^{\boldsymbol{r}}\right)_{a, k}\right)\right. \\
\left.+\sum_{j=1}^{j_{\max }^{2}} \sum_{k=1}^{K_{j}} \Phi_{\eta_{d}^{j}, \lambda_{d}^{j}}^{\beta}\left(\left(F \boldsymbol{s}^{\boldsymbol{r}}\right)_{d, j, k}\right)\right]
\end{array}
$$

where $\eta_{d}^{j} \in \mathbb{C}, \lambda_{d}^{j} \in \mathbb{R}_{+}$and $\beta \geq 1$ (similarly for $\eta_{a}$ and $\left.\lambda_{a}\right)$.

Note that this kind of $\ell_{p}$ regularization has already been successfully used in sparse MRI reconstruction [12].

\section{Optimization procedure}

Based on the formulation above of the spatial and spectral regularization terms (resp. $g(\boldsymbol{S})$ and $h(\boldsymbol{S})$ ), and accounting for the data fidelity term $\mathcal{D}(\boldsymbol{S})=\|\boldsymbol{Z}-\boldsymbol{S}\|_{\Sigma^{-1}}^{2}$, the resulting criterion to be minimized can be written as follows:

$$
\mathcal{J}(\boldsymbol{S})=\mathcal{D}(\boldsymbol{S})+g(\boldsymbol{S})+h(\boldsymbol{S}) .
$$

Note here that if for instance one wants to perform only spatial regularization, the spectral regularization term can be turned off by setting $\lambda_{d}^{j}=\lambda_{a}=0$ in Eq. (3). However, using both regularizations allows eliminating at the same time high spectral and spatial discontinuities.

Since $\mathcal{J}$ is convex, unicity of the target solution is guaranteed. However, $\mathcal{J}$ is not necessarily differentiable, which makes impossible the use of gradient-based algorithms for minimization. We therefore propose to perform the minimization of $\mathcal{J}$ in Eq. (4) by using the concept of proximity operators [13] which was found to be fruitful in a number of recent works in convex optimization [9], [11].

1) Proximity operator of the data fidelity term:

According to standard rules about the calculation of proximity operators the proximity operator of the data fidelity term $\mathcal{D}$ is given by:

$$
\operatorname{prox}_{\mathcal{D}} \boldsymbol{S}=\left(\boldsymbol{I}_{d}+2 \Sigma^{-1}\right)^{-1}\left(\boldsymbol{S}+2 \Sigma^{-1} \boldsymbol{Z}\right) .
$$


2) Proximity operator for the regularization terms: Using the same rules as in Section III-C.1, the proximity operator of $\Phi_{\mu, \alpha}^{p}$ for every $\xi \in \mathbb{C}$ is given by $\operatorname{prox}_{\Phi_{\mu, \alpha}^{p}} \xi=$ $\operatorname{sign}(\xi) \eta$, where $\eta=\eta^{\operatorname{Re}}+\imath \eta^{\operatorname{Im}}$ is the unique solution in $\mathbb{C}_{+}$to $\eta+p \eta^{p-1} \alpha=|\xi-\mu|$. If $p=1$, this proximity operator simplifies as follows:

$$
\begin{aligned}
& \operatorname{prox}_{\Phi_{\mu, \alpha}^{p}} \xi=\operatorname{sign}(\operatorname{Re}(\xi-\mu)) \max \{|\operatorname{Re}(\xi-\mu)|-\operatorname{Re}(\alpha), 0\} \\
& +\imath \operatorname{sign}(\operatorname{Im}(\xi-\mu)) \max \{|\operatorname{Im}(\xi-\mu)|-\operatorname{Im}(\alpha), 0\} .
\end{aligned}
$$

However, due to the spatial and spectral regularization terms in Eqs. (2) and (3), we need to calculate the proximity operators of $\Phi_{\mu, \alpha}^{p} \circ T$ and $\Phi_{\mu, \alpha}^{p} \circ F$, respectively. These operators can be calculated based on [11, Lemma 2.4], leading to $\operatorname{prox}_{\Phi_{\mu, \alpha}^{p} \circ T}=T^{-1} \circ \operatorname{prox}_{\Phi_{\mu, \alpha}^{p} \circ T} \circ T$ (similarly $\operatorname{prox}_{\Phi_{\mu, \alpha}^{p} \circ F}$ ).

3) Optimization algorithm:

Since the cost function in Eq. (4) is made up of more than two non-necessarily differentiable terms, an appropriate solution for its minimization is the Parallel Proximal Algorithm [11]. A key advantage of this algorithm is that its computations can be parallelized while converging to the global minimum. The resulting algorithm for the minimization of the optimality criterion in Eq. (4) is described below.

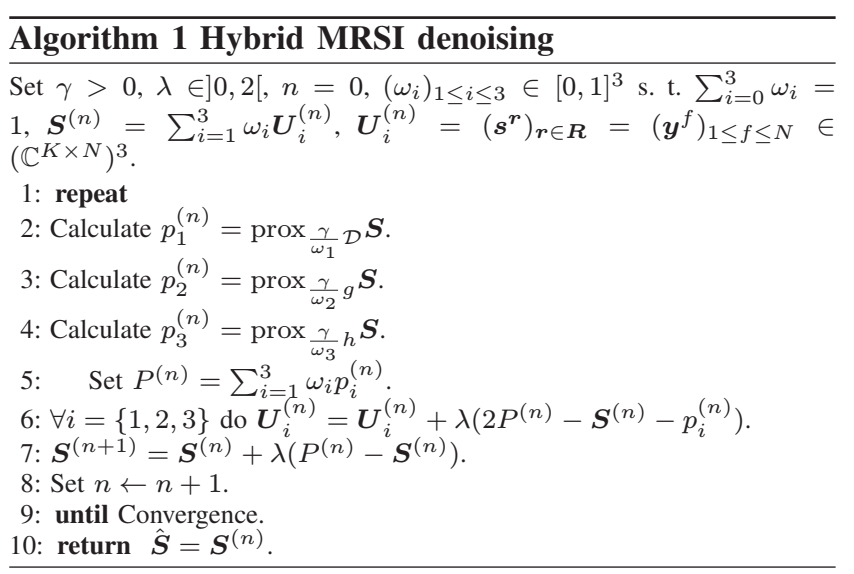

\section{A. Synthetic Data}

\section{Results}

The proposed method was initially tested on synthetic data. We simulated a MRSI data set based on values commonly reported in the literature [14]. Simulated spectra contained the three largest metabolite signals in healthy brain tissue, Choline (Cho), Creatine (Cr) and N-acetyl-aspartate (NAA). Spectral lines were simulated using Gaussian and Lorentzian lineshapes to assess the performance in both cases. Different levels of additive white Gaussian noise were added to the ground truth signal (see Tab. I). The proposed sparse spectral spatial regularization (SSSR) is compared with wavelet shrinkage (WS) and with the spatio-spectral regularization (SSR) proposed in [5]. For each method, regularization parameters have been chosen so as to optimize the output $\mathrm{SNR}^{1}$ while preserving at best the spectral features. It is

$$
{ }^{1} \mathrm{SNR}=20 \log _{10} \frac{\left\|\boldsymbol{S}_{\mathrm{ref}}\right\|_{2}}{\left\|\boldsymbol{S}_{\mathrm{ref}}-\widehat{\boldsymbol{S}}\right\|_{2}}
$$

worth noticing that fully autocalibrated approaches may be used to automatically estimate these parameters directly from the data [15]. SSR showed to be very sensitive to the order and to the prediction coefficients of the Autoregressive model that defines the spectral regularization term. We have chosen here an order $L=3$ to capture the three peaks present in the simulated spectra. Linear prediction coefficients were estimated using a least squares method. In SSSR, $p=\beta=1$ as been used (see Section III-B), and as regards the wavelet transforms, we used a Daubechies basis over one resolution level. Different types of wavelet families were tested and Daubechies showed to be the best suited for this type of data. The size of the images used in the clinical rutine is usually small (no bigger than $32 \times 32$ ). For this reason only one spatial resolution level is considered. Fig. 1 shows the relative concentrations of Cho/NAA obtained from MRSI data using the three different methods. Fig. 2 shows the corresponding error maps $(\sigma=8)$. The noisy map shows fake abnormal voxels (caracterized by Cho/NAA ratio $>2$ ) and voxels where this ratio is understimated. While the other methods only detect a few of these errors, the SSSR is able to correct most of the irregularities and recover values very close to the ground truth. Tab. I gives the SNR values of the denoised MRSI data using the different methods and for different noise levels. The same table also compares the SNR of the Cho/NAA metabolic maps generated with MRSI data denoised using different methods. SNR values reported in Tab. I indicate that SSSR yields improved signal quality when compared to the other approaches. This improvement results in better metabolic maps with SSSR. Regarding computational efficiency, SSSR is computationally more expensive that simple WS since it involves more operations due to the additional spatial term. On the other hand, SSSR showed to be much more efficient than SSR. Indeed, SSSR is 3 times faster than SSR (27 sec in comparison to 80 for the case presented in this experiment). One potential reason could be that SSR implies the inversion of sparse but large matrices.

\section{B. In vivo Data}

To further analyse the robustness of our method, we have tested the results on in vivo MRSI data from human brain. These data were acquired with a Siemens Avanto $1.5 \mathrm{~T}$ using a 3D CSI sequence with water suppression, TE $=135$ $\mathrm{ms}$, TR $=1500 \mathrm{~ms}, 512$ FID data points and 4 averages. Improvements with SSSR, compared to conventional signal apodization, were found on experimental data. Apodization filters tend to broaden resonances and distort spectral lineshapes [4]. By using SSSR, the SNR is increased without decreasing spectral resolution, thus maintaining the spectral features. We have also compared the performance of SSR and SSSR on in vivo MRSI signals. SSSR has shown to be more robust than SSR since it is less sensitive to spectral lineshapes. Fig. 4 shows a real spectrum (related to abnormal tissue) that has been denoised using both methods. We can easily notice that SSSR allows preserving the spectral lineshapes which are under-estimated with SSR. Fig. 3 shows how apodization (Hanning filter) distorts spectral lines while 
they are well preserved after SSSR denoising. Note that for this experiment, the noise covariance matrix $\Sigma$ has been determined using a separated phantom acquisition.
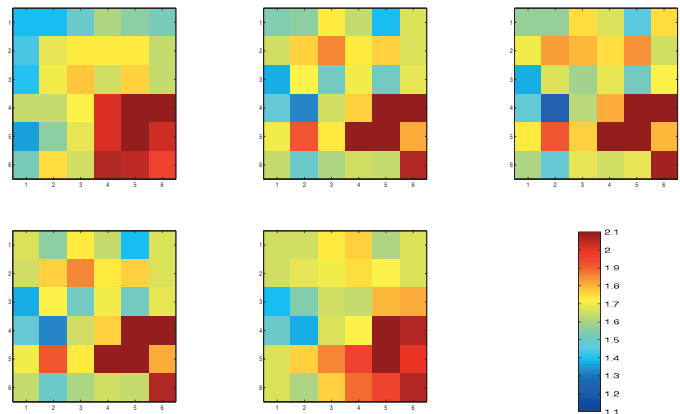

Fig. 1. Cho/NAA metabolic maps based on the ground truth (a), noisy (b) and denoised signals using WS (c), SSR (d) and SSSR (e).

(c) - (a)

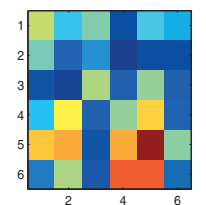

(e) - (a)


Fig. 2. Errors associated with maps of Fig. 1.

TABLE I

SNR $(d B)$ EVALUATION FOR SYNTHETIC DATA DENOISING, BOTH FOR SIGNALS AND CHO/NAA METABOLIC MAPS.

\begin{tabular}{|c|c|c|c|c|c|}
\cline { 3 - 6 } \multicolumn{2}{c|}{} & Noisy & WS & SSR & SSSR \\
\hline \multirow{2}{*}{$\sigma=6$} & Signal & 10.24 & 10.54 & 11.89 & 13.08 \\
\cline { 2 - 6 } & Map & 16.90 & 17.93 & 17.78 & 18.96 \\
\hline \multirow{2}{*}{$\sigma=8$} & Signal & 9.85 & 10.23 & 11.72 & 13.74 \\
\cline { 2 - 6 } & Map & 16.06 & 18.47 & 18.30 & 21.01 \\
\hline \multirow{2}{*}{$\sigma=12$} & Signal & 9.07 & 9.86 & 11.44 & 14.18 \\
\cline { 2 - 6 } & Map & 15.89 & 16.78 & 16.95 & 21.33 \\
\cline { 2 - 6 } & Signal & 8.85 & 10.04 & 11.54 & 14.31 \\
\cline { 2 - 6 } & Map & 15.06 & 16.06 & 16.26 & 18.18 \\
\hline
\end{tabular}

NAA peak

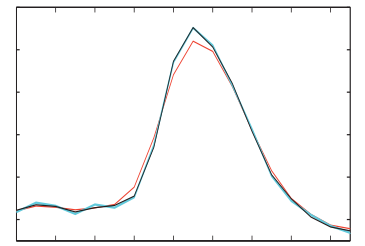

Fig. 3. Comparison of standard apodization and SSSR: raw spectrum (blue), apodized spectrum (red) and spectrum after SSSR denoising (black).

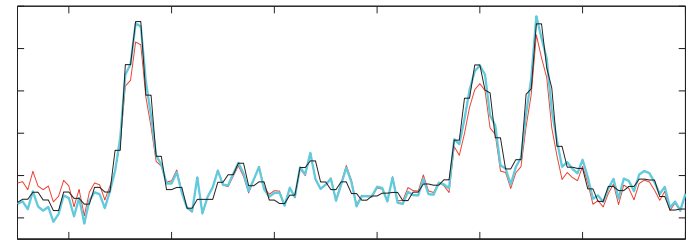

Fig. 4. Spectra denoising comparison: raw spectrum (blue), spectrum after SSR denoising (red) and spectrum after SSSR denoising (black).

\section{CONCLUSion}

We presented a new denoising method for MRSI which overcomes the drawbacks of previous approaches without any lineshape constraint. This property makes our method suitable for handling in vivo data. It explores both spectral and spatial dimensions of MRSI data. The inherent optimization problem is solved using a fast and efficient proximal algorithm that allows parallel computations while guaranteeing the convergence to the global minimum. Furthermore, the proposed approach can be easily extended to incorporate more sophisticated priors, such as tissue distributions and anatomical configuration extracted from companion MRI images. This method will be compared with other promising proposed methods [16], [17] for further validation.

\section{REFERENCES}

[1] A. Laprie, I. Catalaa, E. Cassol, T.R. McKnight, D. Berchery, D. Marre, J.M. Bachaud JM, I. Berry, and E. C. Moyal, "Proton magnetic resonance spectroscopic imaging in newly diagnosed glioblastoma: predictive value for the site of postradiotherapy relapse in a prospective longitudinal study," Int J Radiat Oncol Biol Phys., vol. 70, pp. 773-781, 2008.

[2] L. Kwock, J. K. Smith, M. Castillo, M. G. Ewend, F. Collichio, D. E. Morris, T. W. Bouldin, and S. Cush, "Clinical role of proton magnetic resonance spectroscopy in oncology: brain, breast, and prostate cancer," The Lancet Oncology, vol. 7, pp. 859-868, 2006.

[3] M. G. Kounelakis, M. E. Zervakis, G. J. Postma, L. M. Buydens, A. Heerschap, and X. Kotsiakis, "Revealing the metabolic profile of brain tumors for diagnosis purposes," in IEEE Engineering in Medicine and Biology Society, Sep. 3-6 2009, pp. 35-38.

[4] R. Bartha R, D. J. Drost, and P. C. Williamson, "Factors affecting the quantification of short echo in-vivo $1 \mathrm{H}$ MR spectra: prior knowledge, peak elimination, and filtering," NMR Biomed, vol. 12, pp. 205-216, 1999.

[5] H. M. Nguyen, J. P. Haldar, M. N. Do, and Zhi-Pei Liang, "Denoising of $\mathrm{mr}$ spectroscopic imaging data with spatial-spectral regularization," in IEEE International Symposium on Biomedical Imaging (ISBI), Rotterdam, Netherlands, Apr. 14-17 2010, pp. 720-723.

[6] Y. Bao and A. A. Maudsley, "Improved reconstruction for $\mathrm{mr}$ spectroscopic imaging," IEEE Trans. Med. Imag., vol. 26, pp. 686695, 2007.

[7] A. Diop, Y. Zaim-Wadghiri, A. Briguet, and D. Graveron-Demilly, "Improvements of quantitation by using the cadzow enhancement procedure prior to any linear-prediction methods," J. Magn. Reson., vol. 105, pp. 17-24, 1994.

[8] A. Pizurica, A. M. Wink, E. Vansteenkiste, W. Philips, and J. Roerdink, "A review of wavelet denoising in MRI and ultrasound brain imaging," Current Medical Imaging Reviews, vol. 2, no. 2, pp. 247-260, 2006

[9] L. Chaari, J.-C. Pesquet, A. Benazza-Benyahia, and P. Ciuciu, "A wavelet-based regularized reconstruction algorithm for SENSE parallel MRI with applications to neuroimaging," Med. Image Anal., vol. 15, no. 2, pp. 185-201, 2011.

[10] L. T. Mainardi, D. Origgi, P. Lucia, G. Scotti, and S. Cerutti, "A wavelet packets decomposition algorithm for quantification of in vivo 1H-MRS parameters," Medical Engineering \& Physics, vol. 24, pp. 201-208, 2002.

[11] P. L. Combettes and J.-C. Pesquet, "A proximal decomposition method for solving convex variational inverse problems," Inverse Problems, vol. 24 , no. 6, 2008, 27 p.

[12] L. Chaari, J.-C. Pesquet, A. Benazza-Benyahia, and Ph. Ciuciu, "Autocalibrated parallel MRI reconstruction in the wavelet domain," in Int. Symp. on Biomed. Imag., Paris, France, May 14-17 2008, pp. $756-759$.

[13] J.-J. Moreau, "Proximité et dualité dans un espace hilbertien," Bulletin de la Société Mathématique de France, vol. 93, pp. 273-299, 1965.

[14] V. Govindaraju, K. Young, and A. Maudsley, "Proton NMR chemical shifts and coupling constants for brain metabolites," NMR In Biomedicine, vol. 13, pp. 129-153, 2000.

[15] L. Chaari, J.-C. Pesquet, J.-Y. Tourneret, Ph. Ciuciu, and A. BenazzaBenyahia, "A hierarchical Bayesian model for frame representation," IEEE Trans. on Signal Process., vol. 18, no. 11, pp. 5560-5571, 2010.

[16] O. A. Ahmed, "New denoising scheme for magnetic resonance spectroscopy signals," IEEE Trans. Med. Imag., vol. 24, pp. 809-816, 2005.

[17] H. M. Nguyen, X. Peng, M. N. Do, and Zhi-Pei Liang, "Denoising $\mathrm{mr}$ spectroscopic imaging data with low-rank approximations," IEEE Trans. Biomed. Eng., vol. 60, pp. 78-89, 2013. 